\title{
A Certain Series Associated with Catalan's Constant
}

\author{
V. S. Adamchik
}

Abstract. A parametric class of series generated by integration of complete elliptic integrals $\sum_{-r \neq k=0}^{\infty} \frac{\left(\begin{array}{c}2 k \\ k\end{array}\right)}{(k+r) 16^{k}}$ is valuated in closed form. Alternative proofs to results of Ramanujan and others are given. Also, a particular case of the Saalschützian hypergeometric series ${ }_{4} F_{3}(1)$ is derived.

Keywords: Summation of series, elliptic functions, hypergeometric functions, Catalan's constant

AMS subject classification: Primary 33C, secondary 33E,11Y

\section{Introduction}

The subject of our interest is the hypergeometric series generated by elliptic integrals

$$
S(r)=\sum_{k=0}^{\infty} \frac{\left(\begin{array}{c}
2 k \\
k
\end{array}\right)^{2}}{(k+r) 16^{k}}=\frac{1}{r}{ }_{4} F_{3}\left(\frac{1}{2}, \frac{1}{2}, r ; 1, r+1 ; 1\right) .
$$

This series has a long and interesting story. About a century ago Ramanujan (see [8: p. 351] and [3: p. 39]) in his first letter to Hardy stated without proof a particular case of (1), when the parameter $r=n$ is a positive integer, namely

$$
S(r)=\frac{16^{n}}{\pi n^{2}\left(\begin{array}{c}
2 n \\
n
\end{array}\right)^{2}} \sum_{k=0}^{n-1} \frac{\left(\begin{array}{c}
2 k \\
k
\end{array}\right)^{2}}{16^{k}}
$$

In 1927, when Ramanujan's collected papers were published and result (2) became publicly known, it attracted a great deal of attention. Different proofs were given by Watson [13] and Darling [4], later Bailey [2] and Hodgkinson [9] generalized (2) to

$$
{ }_{3} F_{2}(a, b, c+n-1 ; c, a+b+n ; 1)=\frac{\Gamma(n) \Gamma(a+b+n)}{\Gamma(a+n) \Gamma(b+n)} \sum_{k=0}^{n-1} \frac{(a)_{k}(b)_{k}}{(c)_{k} k !}
$$

V. S. Adamchik: Carnegie Mellon Univ., Dept. Comp. Sci., Pittsburgh, PA 15213-3891, USA; adamchik@cs.cmu.edu 
which gives Ramanujan's result when $a=b=\frac{1}{2}$ and $c=1$. Ramanujan (see [11: pp. 237 - 239] and [3: p. 45]) also stated a complementary formula to (2), when the parameter $r=n+\frac{1}{2}$ is a half integer, namely

$$
S\left(n+\frac{1}{2}\right)=\frac{4}{\pi} \frac{\left(\begin{array}{c}
2 n \\
n
\end{array}\right)^{2}}{16^{n}}\left(2 G+\sum_{k=0}^{n-1} \frac{16^{k}}{\left(\begin{array}{c}
2 k \\
k
\end{array}\right)^{2}(2 k+1)^{2}}\right) .
$$

Here $G$ is Catalan's constant defined by

$$
G=\frac{1}{2} \int_{0}^{1} \mathbf{K}(k) d k=\sum_{k=0}^{\infty} \frac{(-1)^{k}}{(2 k+1)^{2}}
$$

and $\mathbf{K}$ is the complete elliptic integral of the first kind, given by

$$
\mathbf{K}(k)=\int_{0}^{\frac{\pi}{2}} \frac{d t}{\sqrt{1-k^{2} \sin ^{2} t}} .
$$

As mentioned in [3: p. 47], Ramanujan's proofs of formulas (2) and (3) most likely were based on the recurrence equation

$$
\left(r+\frac{1}{2}\right)^{2} S(r+1)-r^{2} S(r)=\frac{1}{\pi}
$$

subject to initial conditions. This equation is derived from the fact that $S(r)$ is generated by integration of complete elliptic integrals as

$$
S(r)=\frac{2}{\pi} \int_{0}^{1} z^{r-1} \mathbf{K}(z) d z \quad(\Re(r)>0) .
$$

In 1981, unawared of Ramanujan's equation (4), Dutka [5] employed (5) to rediscover formulas (2) and (3). In Section 2 we outline the derivation of equation (4), as well as its solution. In view of (4), it is pretty straightforward to see that for any rational $r=n+p$, where $n$ is a positive integer and $0<p \leq 1$, series (1) has a closed form representation

$$
S(n+p)=\frac{(p)_{n}^{2}}{\left(p+\frac{1}{2}\right)_{n}^{2}}\left(S(p)+\frac{1}{\pi p^{2}} \sum_{k=0}^{n-1} \frac{\left(p+\frac{1}{2}\right)_{k}^{2}}{(p+1)_{k}^{2}}\right) .
$$

Here $(p)_{n}=p(p+1) \cdots(p+n-1)$ is the Pochhammer symbol. There are only three known cases when the function $S(p)$ is expressible in terms other than hypergeometric functions, namely $p \in\left\{1, \frac{1}{2}, \frac{1}{4}\right\}$ with

$$
\begin{aligned}
& S(1)={ }_{3} F_{2}\left(\frac{1}{2}, \frac{1}{2}, 1 ; 1,2 ; 1\right)={ }_{2} F_{1}\left(\frac{1}{2}, \frac{1}{2} ; 2 ; 1\right)=\frac{4}{\pi} \\
& S\left(\frac{1}{2}\right)=2_{3} F_{2}\left(\frac{1}{2}, \frac{1}{2}, \frac{1}{2} ; 1, \frac{3}{2} ; 1\right)=\frac{8 G}{\pi} \\
& S\left(\frac{1}{4}\right)=4_{3} F_{2}\left(\frac{1}{2}, \frac{1}{2}, \frac{1}{4} ; 1, \frac{5}{4} ; 1\right)=\frac{\Gamma\left(\frac{1}{4}\right)^{4}}{4 \pi^{2}}
\end{aligned}
$$


where $\Gamma(z)$ is the Euler gamma function. All these cases are due to Ramanujan (see [3]). Glasser [6] made a conjecture that it is possible to express $S\left(\frac{1}{2^{k}}\right)$ for $k \geq 3$ in finite terms, however that is remained to be seen.

It does not appear to have been previously studied the case when the parameter $r$ in (1) is a negative integer (assuming that the term $r=-k$ is dropped from summation):

$$
S(r)=\sum_{-r \neq k=0}^{\infty} \frac{\left(\begin{array}{c}
2 k \\
k
\end{array}\right)^{2}}{(k+r) 16^{k}} .
$$

A few particular cases of (6) appeared in the handbooks by Adams and Hippisley [1] and by Hansen [7]:

$$
\begin{aligned}
& S(-1)=-\frac{2 G+1}{\pi}+\log 2-\frac{1}{2} \\
& S(-2)=-\frac{18 G+13}{16 \pi}+\frac{9}{16} \log 2-\frac{21}{64} .
\end{aligned}
$$

In the present paper, using contour integration technique, we will show that for negative integer $r$ sum (6) is solvable in closed form by

$$
S(r)=-S\left(\frac{1}{2}-r\right)+\frac{4}{16^{-r}}\left(\begin{array}{c}
-2 r \\
-r
\end{array}\right)^{2}\left(H_{-r}-H_{-2 r}+\log 2\right)
$$

where $H_{n}$ are the harmonic numbers $H_{n}=\sum_{k=1}^{n} \frac{1}{k}$.

As a consequence of this result, in Section 3 we derive the new representation for Saalschẗzian ${ }_{4} F_{3}(1)$ series with a special set of the parameters

$$
\begin{aligned}
& \left(n-\frac{1}{2}\right)_{4} F_{3}\left(1,1, n+\frac{1}{2}, n+\frac{1}{2} ; 2, n+1, n+1 ; 1\right) \\
& \quad=\frac{4 n^{2}}{2 n-1}\left(H_{n-1}+\log 4\right)-\frac{16^{n}}{\left(\begin{array}{c}
2 n \\
n
\end{array}\right)^{2}}{ }_{3} F_{2}\left(\frac{1}{2}, \frac{1}{2}, n-\frac{1}{2} ; 1, n+\frac{1}{2} ; 1\right) .
\end{aligned}
$$

\section{Evaluation}

We consider two cases, namely when $r$ is positive and negative. We denote

$$
\begin{array}{ll}
S^{+}(r)=S(r) & (\Re(r)>0) \\
S^{-}(r)=S(r) & (\Re(r) \leq 0) .
\end{array}
$$

Let $r$ be a positive integer. We transform series (1) to a definite integral involving complete elliptic integrals. Multiplying the summand by $x^{k+r}$ and differentiating it with respect to $x$, we get

$$
g(r, x)=x^{r-1} \sum_{k=0}^{\infty}\left(\begin{array}{c}
2 k \\
k
\end{array}\right)^{2} \frac{x^{k}}{16^{k}}=\frac{2}{\pi} x^{r-1} \mathbf{K}(x)
$$

for $|x|<1$ where $\mathbf{K}(x)$ is the elliptic integral. Integrating both sides of (7), we arrive at

$$
S^{+}(r)=\int_{0}^{1} g(r, x) d x=\frac{2}{\pi} \int_{0}^{1} x^{r-1} \mathbf{K}(x) d x \quad(\Re(r)>0) .
$$


In the next subsections we evaluate $S^{+}(r)$ by first developing a recurrent equation for $S^{+}(r)$ and then solving it by iteration. The result depends on the disparity of $r$.

Now let us consider the second case when $r$ is a negative integer. We split the series $S(r)$ into two sums as

$$
S^{-}(r)=\sum_{-r \neq k=0}^{\infty} \frac{\left(\begin{array}{c}
2 k \\
k
\end{array}\right)^{2}}{(k+r) 16^{k}}=\left(\sum_{k=0}^{-r-1}+\sum_{k=-r+1}^{\infty}\right) \frac{\left(\begin{array}{c}
2 k \\
k
\end{array}\right)^{2}}{(k+r) 16^{k}} .
$$

Leaving the first sum unchanged, and converting the second sum into an elliptic integral (by applying the same reasoning as above), we obtain

$$
S^{-}(r)=\sum_{k=0}^{-r-1} \frac{\left(\begin{array}{c}
2 k \\
k
\end{array}\right)^{2}}{(k+r) 16^{k}}+\int_{0}^{1} x^{r-1}\left(\frac{2}{\pi} \mathbf{K}(x)-\sum_{k=0}^{-r}\left(\begin{array}{c}
2 k \\
k
\end{array}\right)^{2} \frac{x^{k}}{16^{k}}\right) d x
$$

for $\Re(r) \leq 0$. In Subsection 2.3, using contour integration technique, we establish a functional relation transforming $S^{-}(r)$ into $S^{+}(r)$.

2.1 $S^{+}(r)$ for $r$ a non-negative integer. Consider the system of indefinite integrals

$$
\left.\begin{array}{l}
k_{p}(x)=\int x^{p} \mathbf{K}(x) d x \\
e_{p}(x)=\int x^{p} \mathbf{E}(x) d x
\end{array}\right\}
$$

where the parameter $p$ is a positive integer or zero, and $\mathbf{E}(x)$ and $\mathbf{K}(x)$ are complete elliptic integrals. Using integration by parts, the above integral system can be reduced to the system of coupled recurrent equations

$$
\left.\begin{array}{l}
k_{p}(x)=x^{p} k_{0}(x)-2 p\left(k_{p}(x)-k_{p-1}(x)+e_{p-1}(x)\right) \\
e_{p}(x)=x^{p} e_{0}(x)-\frac{2}{3} p\left(e_{p-1}(x)+e_{p}(x)+k_{p}(x)-k_{p-1}(x)\right)
\end{array}\right\}
$$

with initial conditions

$$
\begin{aligned}
& 2 k_{0}(x)=\mathbf{E}(x)+(x-1) \mathbf{K}(x) \\
& \frac{3}{2} e_{0}(x)=(x+1) \mathbf{E}(x)+(x-1) \mathbf{K}(x) .
\end{aligned}
$$

Eliminating $e_{p-1}(x)$ from the first equation, and $k_{p-1}(x)$ and $k_{p}(x)$ from the second, the system is simplified to

$$
\left.\begin{array}{rl}
k_{p}(x)= & \frac{4 p^{2}}{(2 p+1)^{2}} k_{p-1}(x)+\frac{2 x^{p} \mathbf{E}(x)+2(2 p+1)(x-1) x^{p} \mathbf{K}(x)}{(2 p+1)^{2}} \\
e_{p}(x)= & \frac{4 p^{2}}{(2 p+1)(2 p+3)} e_{p-1}(x) \\
& +\frac{2(1-2 p+(2 p+1) x) x^{p} \mathbf{E}(x)+2(x-1) x^{p} \mathbf{K}(x)}{(2 p+1)(2 p+3)}
\end{array}\right\} .
$$


Now we compute the values of $k_{p}(x)$ and $e_{p}(x)$ at the limiting points $x=0$ and $x=1$. We get two recurrent equations

$$
\left.\begin{array}{l}
k_{p}(0)=0 \quad(p \geq 0) \\
k_{0}(1)=2 \\
k_{p}(1)=\frac{4 p^{2}}{(2 p+1)^{2}} k_{p-1}(1)+\frac{2}{(2 p+1)^{2}} \quad(p \geq 1)
\end{array}\right\}
$$

and

$$
\begin{aligned}
& e_{p}(0)=0 \quad(p \geq 0) \\
& e_{p}(1)=\frac{4 p^{2}}{(2 p+1)(2 p+3)} e_{p-1}(1)+\frac{4}{(2 p+1)(2 p+3)} \quad(p \geq 1) .
\end{aligned}
$$

In view of formulas (8) and (11) we conclude that

$$
S^{+}(r)=\frac{2}{\pi}\left(k_{r-1}(1)-k_{r-1}(0)\right)=\frac{2}{\pi} k_{r-1}(1)
$$

where $S^{+}(r)$ satisfies the recurrence relation

$$
\left.\begin{array}{rl}
S^{+}(1) & =\frac{4}{\pi} \\
\left(r+\frac{1}{2}\right)^{2} S^{+}(r+1)-r^{2} S^{+}(r) & =\frac{1}{\pi} \quad(r \geq 1)
\end{array}\right\} .
$$

This recurrence equation can be solved by iteration (see Section 4 for details).

We have proven

Proposition 2.1. Let $n$ be a positive even. Then $S(n)$ defined by (1) evaluates to

$$
S(n)=\frac{16^{n}}{\pi n^{2}\left(\begin{array}{c}
2 n \\
n
\end{array}\right)^{2}} \sum_{k=0}^{n-1}\left(\begin{array}{c}
2 k \\
k
\end{array}\right)^{2} \frac{1}{16^{k}} .
$$

2.2 $S^{+}(r)$ for $\boldsymbol{r}$ a positive half-integer. Consider slightly different (than (10)) system of indefinite integrals

$$
\left.\begin{array}{rl}
\widehat{k}_{p}(x) & =\int x^{p-\frac{1}{2}} \mathbf{K}(x) d x \\
\widehat{e}_{p}(x) & =\int x^{p-\frac{1}{2}} \mathbf{E}(x) d x
\end{array}\right\}
$$

where the parameter $p$ is a positive integer or zero, and $\mathbf{E}(x)$ and $\mathbf{K}(x)$ are complete elliptic integrals. Using integration by parts, we transform (13) to the system of recurrent equations

$$
\begin{aligned}
p^{2} \widehat{k}_{r}(x) & =\left(p-\frac{1}{2}\right)^{2} \widehat{k}_{p-1}(x)+\frac{1}{2} x^{p-\frac{1}{2}}(\mathbf{E}(x)+2 p(x-1) \mathbf{K}(x)) \\
p(p+1) \widehat{e}_{r}(x) & =\left(p-\frac{1}{2}\right)^{2} \widehat{e}_{p-1}(x)+x^{p-\frac{1}{2}}\left((p(x-1)+1) \mathbf{E}(x)+\frac{x-1}{2} \mathbf{K}(x)\right)
\end{aligned}
$$


where

$$
\begin{aligned}
& \widehat{k}_{0}(x)=\pi \sqrt{x}_{3} F_{2}\left(\frac{1}{2}, \frac{1}{2}, \frac{1}{2} ; 1, \frac{3}{2} ; x\right) \\
& \widehat{e}_{0}(x)=\pi \sqrt{x}_{3} F_{2}\left(-\frac{1}{2}, \frac{1}{2}, \frac{1}{2} ; 1, \frac{3}{2} ; x\right)
\end{aligned}
$$

and ${ }_{3} F_{2}(x)$ is the hypergeometric function. By computing the limits at $x=0$ and $x=1$, system (14) yields

$$
\begin{aligned}
& \widehat{k}_{p}(0)=0 \quad(p \geq 0) \\
& \widehat{k}_{0}(1)=4 G \\
& \widehat{k}_{p}(1)=\frac{\left(p-\frac{1}{2}\right)^{2}}{p^{2}} \widehat{k}_{p-1}(1)+\frac{1}{2 p^{2}} \quad(p \geq 1)
\end{aligned}
$$

where G is Catalan's constant. Therefore, $S^{+}\left(p+\frac{1}{2}\right)=\frac{2}{\pi} \widehat{k}_{p}(1)(p \geq 0)$. The sequence $S^{+}(r)$, where $r$ is a positive half integer, satisfies the same recurrence equation (12), but with a different initial condition

$$
\begin{aligned}
S^{+}\left(\frac{1}{2}\right) & =\frac{8 G}{\pi} \\
\left(r+\frac{1}{2}\right)^{2} S^{+}(r+1)-r^{2} S^{+}(r) & =\frac{1}{\pi} .
\end{aligned}
$$

Solving this recurrence by iteration (see Section 4 for details), we have proven

Proposition 2.2. Let $n$ be a positive integer. Then $S\left(n+\frac{1}{2}\right)$ defined by (1) evaluates to

$$
S\left(n+\frac{1}{2}\right)=\frac{4}{\pi} \frac{\left(\begin{array}{c}
2 n \\
n
\end{array}\right)^{2}}{16^{n}}\left(2 G+\sum_{k=0}^{n-1} \frac{16^{k}}{\left(\begin{array}{c}
2 k \\
k
\end{array}\right)^{2}(2 k+1)^{2}}\right) .
$$

$2.3 \boldsymbol{S}^{-}(\boldsymbol{r})$ for $\boldsymbol{r}$ a negative integer. Recall formula (9). Observing that the finite sum inside of the integrand $\sum_{k=0}^{-r}\left(\begin{array}{c}2 k \\ k\end{array}\right)^{2} \frac{x^{k}}{16^{k}}$ is the Taylor expansion of $\frac{2}{\pi} \mathbf{K}(x)$ at $x=0$, we pull that sum out of integration, by understanding integration in the Hadamard sense (finite part). Computing limits at the end points and obliterating logarithmic and polynomial order singularities, we get

$$
S^{-}(r)=\text { f.p. } \frac{2}{\pi} \int_{0}^{1} x^{-r-1} \mathbf{K}(x) d x .
$$

Comparing this integral with formula (8) immediately implies that

$$
S^{-}(r)=S^{+}(r)+F(r)
$$

where $F(r)$ is an unknown function. The necessity of $F$ becomes obvious once we recall that in the original series we skip the term $k=-r$, when $r$ is a negative integer. In order to find $F$, we derive a contour integral representation for the sum $S(r)$ as

$$
S(r)=\frac{1}{2 \pi i} \int_{\gamma-i \infty}^{\gamma+i \infty} \frac{\Gamma(s) \Gamma\left(\frac{1}{2}-s\right)}{\Gamma(1-s) \Gamma\left(\frac{1}{2}+s\right)} \frac{d s}{r-s} .
$$


The contour $(\gamma-i \infty, \gamma+i \infty)$ is a straight line lying in the strip $0<\gamma=\Re(s)<\frac{1}{2}$. In fact, evaluating integral (17) by residues at single poles $s=0,-1,-2, \ldots$, lying to the left of the contour, we arrive at series (1). However, if $r$ is a negative integer, the integrand in (17) has a double pole at $s=r$. According to the definition of $S^{-}(r)$ we must skip this pole. Thus, we have

$$
\begin{aligned}
S^{-}(r)= & \frac{1}{2 \pi i} \int_{\gamma-i \infty}^{\gamma+i \infty} \frac{\Gamma(s) \Gamma\left(\frac{1}{2}-s\right)}{\Gamma(1-s) \Gamma\left(\frac{1}{2}+s\right)} \frac{d s}{r-s} \\
& -\operatorname{res}_{s=r}\left(\frac{\Gamma(s) \Gamma\left(\frac{1}{2}-s\right)}{\Gamma(1-s) \Gamma\left(\frac{1}{2}+s\right)} \frac{1}{r-s}\right) .
\end{aligned}
$$

As a matter of fact, the contour integral herein can also be computed via residues at the poles $s=\frac{1}{2}, \frac{3}{2}, \ldots$, lying to the right of the contour. Evaluating the integral via those poles allows us to avoid the double pole at $s=r$. This yields

$$
\begin{aligned}
\frac{1}{2 \pi i} \int_{\gamma-i \infty}^{\gamma+i \infty} \frac{\Gamma(s) \Gamma\left(\frac{1}{2}-s\right)}{\Gamma(1-s) \Gamma\left(\frac{1}{2}+s\right)} \frac{d s}{r-s} & =-\sum_{k=0}^{\infty} \frac{(2 k) !^{2}}{k !^{4}\left(k-r+\frac{1}{2}\right) 16^{k}} \\
& =-S^{+}\left(\frac{1}{2}-r\right) .
\end{aligned}
$$

Finally, computing the residue

$$
\operatorname{res}_{s=r}\left(\frac{\Gamma(s) \Gamma\left(\frac{1}{2}-s\right)}{\Gamma(1-s) \Gamma\left(\frac{1}{2}+s\right)} \frac{1}{r-s}\right)=\frac{4}{16^{-r}}\left(\begin{array}{c}
-2 r \\
-r
\end{array}\right)^{2}\left(H_{-2 r}-H_{-r}-\log 2\right)
$$

we establish

Proposition 2.3. Let $r$ be a negative integer or zero. Then

$$
S^{-}(r)=-S^{+}\left(\frac{1}{2}-r\right)-\frac{4}{16^{-r}}\left(\begin{array}{c}
-2 r \\
-r
\end{array}\right)^{2}\left(H_{-r}-H_{-2 r}+\log 2\right)
$$

where $S^{+}\left(\frac{1}{2}-r\right)$ is defined in Proposition 2.2 .

$2.4 S^{-}(r)$ for $r$ a negative half integer. This case immediately follows from the previous subsection, taking into consideration that the integrand in (17) has only a single pole at $s=r$.

Proposition 2.4. Let $n$ be a positive integer. Then $S^{-}\left(-n+\frac{1}{2}\right)=-S^{+}(n)$. 


\section{Special cases of hypergeometric functions}

In this section we derive a particular case of the Saalschützian hypergeometric series ${ }_{4} F_{3}(1)$. We begin by recalling that the hypergeometric series

$$
{ }_{p+1} F_{p}\left(a_{1}, \ldots, a_{p+1} ; b_{1}, \ldots, b_{p} ; 1\right)
$$

is called Saalschützian if the parameters $a_{i}$ and $b_{i}$ satisfy the relation

$$
1+a_{1}+\ldots+a_{p+1}=b_{1}+\ldots+b_{p}
$$

Proposition 3.1. Let $n$ be a positive integer. Then

$$
\begin{aligned}
& \frac{(2 n-1)^{2}}{8 n^{2}}{ }_{4} F_{3}\left(1,1, n+\frac{1}{2}, n+\frac{1}{2} ; 2, n+1, n+1 ; 1\right) \\
& =-\frac{4 G}{\pi}+H_{n-1}+\log 4-\frac{2}{\pi} \sum_{k=0}^{n-2} \frac{16^{k}}{(2 k+1)^{2}\left(\begin{array}{c}
2 k \\
k
\end{array}\right)^{2}}
\end{aligned}
$$

where $G$ is Catalan's constant and $H_{n}$ are harmonic numbers.

Proof. In view of formula (18) with $r=-n \quad\left(n \in \mathbb{N}_{0}\right)$ we have

$$
S^{-}(-n)=-S^{+}\left(n+\frac{1}{2}\right)-\frac{4}{16^{n}}\left(\begin{array}{c}
2 n \\
n
\end{array}\right)^{2}\left(H_{n}-H_{2 n}+\log 2\right)
$$

where $S^{+}\left(n+\frac{1}{2}\right)$ is defined in (16). On the other hand, if we evaluate the original sum (6) by means of the hypergeometric function, we obtain

$$
\begin{aligned}
S^{-}(-n)= & \sum_{k=0}^{n-1} \frac{\left(\begin{array}{c}
2 k \\
k
\end{array}\right)^{2}}{(k-n) 16^{k}} \\
& +\frac{\left(\begin{array}{c}
2 n+2 \\
n+1
\end{array}\right)^{2}}{16^{n+1}} F_{3}\left(1,1, n+\frac{3}{2}, n+\frac{3}{2} ; 2, n+2, n+2 ; 1\right) .
\end{aligned}
$$

The finite sum in the right-hand side herein can be evaluated in terms of harmonic numbers (see Proposition 4.2) as

$$
16^{n} \sum_{k=0}^{n-1} \frac{\left(\begin{array}{c}
2 k \\
k
\end{array}\right)^{2}}{16^{k}(n-k)}=4\left(\begin{array}{c}
2 n \\
n
\end{array}\right)^{2} \sum_{k=0}^{n-1} \frac{1}{2 k+1}=2\left(\begin{array}{c}
2 n \\
n
\end{array}\right)^{2}\left(2 H_{2 n-1}-H_{n-1}\right) .
$$

Combining formulas (20) and (21), and replacing $n$ by $n-1$, we arrive at (19)

Remark 3.2. By using different ideas, formula (19) was first proved in [10]. 


\section{Addendum}

In this section we provide a solution to equations (12) and (15).

Proposition 4.1. The solution to the recurrence relation

$$
\left.\begin{array}{rl}
x_{1} & =b \\
(2 n+1)^{2} x_{n+1}-(2 n)^{2} x_{n} & =a \quad(n \geq 1)
\end{array}\right\}
$$

$i s$

$$
x_{n}=\frac{16^{n}}{4 n^{2}\left(\begin{array}{c}
2 n \\
n
\end{array}\right)^{2}}\left(b+a \sum_{k=1}^{n-1} \frac{\left(\begin{array}{c}
2 k \\
k
\end{array}\right)^{2}}{16^{k}}\right) .
$$

Proof. We solve the recurrence by iteration. Iterating it $n-1$ times, we get

$$
x_{n+1}=b \prod_{j=0}^{n-1} \frac{(2 n-2 j)^{2}}{(2 n-2 j+1)^{2}}+a \sum_{k=0}^{n-1} \frac{\prod_{j=0}^{k-1}(2 n-2 j)^{2}}{\prod_{j=0}^{k}(2 n+1-2 j)^{2}} .
$$

In pretty straightforward manner the finite products herein can be converted to the binomial coefficients by using Euler's product representation for the Gamma function. We obtain

$$
\prod_{j=0}^{n-1} \frac{(2 n-2 j)}{(2 n-2 j+1)}=\frac{4^{n+1}}{2(n+1)\left(\begin{array}{c}
2 n+2 \\
n+1
\end{array}\right)}
$$

and

$$
\sum_{k=0}^{n-1} \frac{\prod_{j=0}^{k-1}(2 n-2 j)^{2}}{\prod_{j=0}^{k}(2 n-2 j+1)^{2}}=\frac{16^{n+1}}{4(n+1)^{2}\left(\begin{array}{c}
2 n+2 \\
n+1
\end{array}\right)^{2}} \sum_{k=1}^{n} \frac{\left(\begin{array}{c}
2 k \\
k
\end{array}\right)^{2}}{16^{k}} .
$$

Substituting them into (22) yields the desired result

Proposition 4.2. Let $n$ be a positive integer. Then

$$
\frac{16^{n}}{4\left(\begin{array}{c}
2 n \\
n
\end{array}\right)^{2}} \sum_{k=0}^{n-1} \frac{\left(\begin{array}{c}
2 k \\
k
\end{array}\right)^{2}}{16^{k}(n-k)}=\sum_{k=0}^{n-1} \frac{1}{2 k+1} .
$$

Proof. We rearrange the terms in the sum in the left-hand side of (23) by summing them in the opposite order from $n-1$ to 0 . We get

$$
\sum_{k=0}^{n-1} \frac{\left(\begin{array}{c}
2 k \\
k
\end{array}\right)^{2}}{(n-k) 16^{k}}=\sum_{k=1}^{n} \frac{\left(\begin{array}{c}
2 n-2 k \\
n-k
\end{array}\right)^{2}}{k 16^{n-k}} .
$$

Since the summand evaluates to zero for $k>n$, we extend the range of summation to infinity. Using the definition of the hypergeometric series, we rewrite that sum in terms of ${ }_{4} F_{3}$ as

$$
\frac{16^{n}}{4\left(\begin{array}{c}
2 n \\
n
\end{array}\right)^{2}} \sum_{k=1}^{\infty} \frac{\left(\begin{array}{c}
2 n-2 k \\
n-k
\end{array}\right)^{2}}{k 16^{n-k}}=\frac{n^{2}}{(2 n-1)^{2}}{ }_{4} F_{3}\left(1,1,1-n, 1-n ; 2, \frac{3}{2}-n, \frac{3}{2}-n ; 1\right) .
$$


The latter further simplifies to polygamma functions by [13: Formula 7.5.3.43] as

$$
\begin{aligned}
\frac{2 n^{2}}{(2 n-1)^{2}}{ }_{4} F_{3}\left(1,1,1-n, 1-n ; 2, \frac{3}{2}-n, \frac{3}{2}-n ; 1\right) & =\psi\left(n+\frac{1}{2}\right)-\psi\left(\frac{1}{2}\right) \\
& =\sum_{k=0}^{n-1} \frac{2}{2 k+1}
\end{aligned}
$$

and the statement is proven

Acknowledgment. I would like to thank S. Finch, L. Glasser and R. Richberg for discussions and help regarding references. This work was supported, in part, by NFS grant CCR-0204003.

\section{References}

[1] Adams, E. P. and R. L. Hippisley: Smithsonian Mathematical Formulae and Tables of Elliptic Functions, 3d reprint (Smithsonian miscellaneous collections: Vol. 74/No. 1.). Washington: Smithsonian Inst. 1957.

[2] Bailey, W. N.: The partial sum of the coefficients of the hypergeometric series. J. London Math. Soc. 6 (1931), $40-41$.

[3] Berndt, B.: Ramanujan's Notebooks, Vol.2. New York: Springer-Verlag 1989.

[4] Darling, H. B. C.: On a proof of one of Ramanujan's theorems. J. London Math. Soc. 5 (1930), $8-9$.

[5] Dutka, J.: Two results of Ramanujan. SIAM J. Math. Anal. 12 (1981), $471-476$.

[6] Glasser, L.: Private communication.

[7] Hansen, E. R.: A Table of Series and Products. Englewood Cliffs (NJ, USA): PrenticeHall, 1975.

[8] Hardy, G. H. et al.: Collected Papers of Srinivasa Ramanujan. New York: Chelsea 1962.

[9] Hodgkinson, J.: Note on one of Ramanujan's theorem. J. London Math. Soc. 6 (1931), $42-43$.

[10] Montaldi, E. and G. Zucchelli: Some formulas of Ramanujan, revisited. Siam. J. Math. Anal. 23 (1992), $562-569$.

[11] Notebooks of Srinivasa Ramanujan, Vol. 1. Bombay: Tata Inst. Fund. Res. 1957.

[12] Prudnikov, A. P., Brychkov, Yu. A. and O. I. Marichev: Integrals and Series:More Special Functions. New York: Gordon and Breach 1989.

[13] Watson, G. N.: Theorems stated by Ramanujan (VIII): theorems on divergent series. J. London Math. Soc. 4 (1929), $82-86$.

Received 11.07.2001; in revised form 15.05.2002 